\title{
Impacts of Nanoparticles and Nano Rod Arrays on Optical Generation Rate in Plasmonic-Based Solar Cells
}

\author{
Farshad Farhadnia*, Ali Rostami, and Samieh Matloub \\ Faculty of Electrical and Computer Engineering, University of Tabriz, Tabriz, Iran
}

*Corresponding Author Email: farhadnia@tabrizu.ac.ir

Received: Sep. 1, 2016, Revised: Sep. 28, 2016, Accepted: Oct. 17, 2016, Available Online: Aug. 8, 2017

DOI: 10.18869 /acadpub.ijop.11.2.103

\begin{abstract}
In this article, the effect of plasmonics properties of metal nanorods and nanoparticles on solar cell performance were investigated and simulated. Due to the classic solar cell disadvantages, it seems that a plasmonic solar cell is one of these methods. In plasmonic solar cells, because of plasmonic effect, a high electric field builds around metal nanoparticles so that high conversion efficiency is available. In this study, it is shown that the near-field electromagnetic wave severely affects the generation rate, which handles the carrier's generation in the solar cell equations. By manipulating the plasmonic properties of nanoparticles or nanorods in solar cells structure, distribution of the electromagnetic fields are altered. In this work, optical power and generation rate related to the poynting vector is calculated. So, for improving the generation rate as an important parameter in solar cells, the alteration of nanoparticles or nanorods material, shape, inter-distance between them and medium material, are done. Finally, the comparison between classical solar cell and our improved structure is performed.
\end{abstract}

KEYWORDS: Solar cells, plasmonic, Nanorod, nanoparticle.

\section{I.INTRODUCTION}

Photovoltaic (PV), which contains the direct conversion of sunlight into electric energy, is one component of an evolving energy mix. Several attractive features of this technology include pollution-free operation, relatively low costs and modularity. In future, energy solar cells may well occupy an increasingly prominent role in global electricity generation in order to limit environmental pollution and to slow down the rate of greenhouse gas concentration's rise [1-5]. As a simple model, a semiconductor solar cell is based on a simple p-n junction. A qualitative description of cell performance can be given in terms of a simple model based on the Shockley diode equation in the dark and under illumination. This model is sufficient for understanding the basic carrier transport mechanisms in the cell, and roughly for predicting the performance parameters of a solar cell. It has been reported that most of the traditional solar cells are based on silicon because of its stability, non-toxicity, welldeveloped technology and more abundance in nature. However, a major limitation if thinfilm solar cells are the poor absorption of light, as compared to wafer-based solar cells [6-10]. It is well established that light-trapping by increasing its optical path length inside the absorbing film, for obtaining high efficiency, is crucial for thin-film solar cells. In light of the above restriction, a new method using plasmonic structures can improve the absorption of light, due to excitation of localized surface plasmon. Noble metals such as silver $(\mathrm{Ag})$, gold $(\mathrm{Au})$, aluminum $(\mathrm{Al})$ and copper $(\mathrm{Cu})$, support surface plasmon due to their free electron like behavior. Surface 
plasmon is the collective oscillations of excited free electrons of metallic particles. This unique property of metallic nanoparticles (NPs) can be used to enhance the optical absorption of solar cells through scattering of light and near-field concentration of light [1117]. The contribution of these mechanisms depends on the particle material, shape, size and refractive index of the surrounding dielectric. Hence, the optical power and generation rate related to the poynting vector is calculated. So, in this work, for improving the generation rate as an important parameter in solar cells the alteration of nanoparticles or Nanorods materials, shapes, inter-distance between them and medium material, were done. Finally, the comparison between traditional solar cell and our improved structure was performed. At last, it should be noted that all of the simulation in this work was done by COMSOL 4.4 and MATLAB.

\section{BACKGROUND AND THEORY}

A few equations describe the behavior of charge carriers in semiconductors under the influence of an electromagnetic field; all of which cause deviations from thermal equilibrium conditions. These equations are called the basic equations for semiconductor device. In the following one direction formula, the Poisson equation relates the static electric field to the space-charge density [2-4]:

$$
\frac{d^{2} \varphi(x)}{d x^{2}}=-\frac{d \varepsilon(x)}{d x}=-\frac{\rho(x)}{\varepsilon_{0} \varepsilon_{\mathrm{s}}}
$$

where $\varphi, \varepsilon_{0}$, and $\varepsilon_{s}$ are the electrostatic potential, the permittivity of free space and the static relative permittivity of the medium, respectively. Based on our study, the electron current density $\left(J_{e}\right)$ and the hole current density $\left(J_{h}\right)$ are given by:

$$
\begin{aligned}
& J_{e}(x)=+q D_{e} \frac{d n(x)}{d x}+q \mu_{e} \varepsilon(x) n(x) \\
& J_{h}(x)=+q D_{h} \frac{d p(x)}{d x}+q \mu_{h} \varepsilon(x) p(x)
\end{aligned}
$$

where $p, \mu_{e}, \mu_{e}, D_{e}$, and $D_{h}$ are electron and hole densities, the electron and hole motilities, and the electron and hole diffusion constants, respectively. It is notable that the first terms on the right hand side of Eqs. 2.2 and 2.3 are diffusion currents driven by a concentration gradient, and the second terms are drift currents driven by the electric field. Indeed, the latter case will be manipulated by nanoparticles' (NPs) or nanorods' (NRs) plasmonic radiation. It means that by the use of NPs/NRs, electromagnetic distribution in the different sections of $\mathrm{p}-\mathrm{n}$ junction will be altered, so the drift currents driven by the electric field will be changed. Also, by changing the NPs' materials, shape, geometry and inter-distance between them, the electric field distribution will be easily manipulated. In the following formula, divergence of the current density, $J, \quad$ is contributed to recombination and generation rates of charge carriers by the continuity equation. Electron and hole continuity equations may be written as:

$$
\begin{aligned}
& \frac{1}{q} \frac{d J_{e}(x)}{d x}-r_{e}(x)+G_{e}(x)=0 \\
& \frac{1}{q} \frac{d J_{h}(x)}{d x}+r_{h}(x)-G_{h}(x)=0
\end{aligned}
$$

In these equations $\mathrm{r}(\mathrm{x})$ and $\mathrm{G}(\mathrm{x})$ are the position-dependent volume recombination and photo-generation rates, respectively. Finally, by substituting Eqs 2 and 3 into the continuity equations, Eqs 4 and 5, a couple set of transport equations are driven as:

$$
\begin{aligned}
& D_{e} \frac{d^{2} n}{d x^{2}}+\mu_{e} \varepsilon \frac{d n}{d x}+n \mu_{e} \frac{d \varepsilon}{d x}-r_{e}(x)+G_{e}(x)=0 \\
& D_{h} \frac{d^{2} \rho}{d x^{2}}+\mu_{h} \varepsilon \frac{d \rho}{d x}+\rho \mu_{h} \frac{d \varepsilon}{d x}-r_{h}(x)+G_{h}(x)=0
\end{aligned}
$$

Electron and hole transport equations, Eqs 6 and 7 , are coupled by the electric field E. The coupled set of differential equations, Eqs 1, 6 and 7, can be solved with different degrees of accuracy. It is important to note that for improving the solar cells' specification, in this 
work, we used NPs plasmonic properties, which influence the original electric field in near-field state. In other words, the original field in the solar cell structure will be perturbed by NPs plasmonic in near-field. For extra and effective manipulation, arrays of NPs or NRs was used. It is known that because of the interaction between NPs plasmonics, non-uniform electromagnetic fields are arisen in solar cell's different areas, which contributes to optical power and optical generation rate. Actually, optical generation rate as an important parameter in the solar cell system, contributes to the time averaged Poynting vector, which is given by [6]:

$G_{o p t}=-\frac{\eta_{o p t}}{\hbar v} \nabla \cdot \mathbf{S}_{a v e}$

where, $\eta_{\text {opt }}, S_{\text {ave }}, \hbar$ and optical quantum yield, time averaged Poynting vector, Planck constant and incidence frequency, respectively. The time averaged Poynting vector has been considered as the radiant flux around any NPs and calculated by $\mathrm{E} \times \mathrm{H}^{*}$.The optical generation rate in Eq. 8 can be substituted in Eqs. 4 and 5 and electron and hole continuity equations can be re-written by this alteration. Indeed, by manipulating optical generation rate in Eq. 8, the solar cell's key parameters such as photon current will be changed. The improved solar cell photon current can be illustrated as $[18,19]$ :

$I_{p h}=\left[I_{p h s}+k_{i}\left(T_{c}-T_{r}\right)\right] \frac{G}{G_{r e f}}$,

$G=G_{\text {incidence }}+G_{\text {plasmonic }}$

In this equation, $\mathrm{I}_{p h s}, k_{i}, T_{c}, T_{r}$ are solar cell current under standard condition, temperature coefficient of short circuit current, solar cell operating temperature and reference temperature, respectively. Moreover, $G$ and $G_{\text {ref }}$ contribute to the intensity of irradiance in solar cell and reference intensity, about $10^{3}$ Watts $/ \mathrm{m}^{2}$, respectively. As a result of that $\mathrm{G}$ include the incidence irradiance $\left(G_{\text {incidence }}\right)$ as well as intensity generated by the NPs nearfield plasmonic field $\left(G_{\text {plasmonic }}\right)$. This case shows that the plasmonic field severely enhances the solar cell outputs such as photocurrent and $\mathrm{G}$ is comparable and may be larger than the classical version [1-2, 13]. It should be noted that after calculating solar cell photon current, the solar cell open circuit voltage and short circuit current can be obtained, which are given by:

$V_{o c}=\frac{A K T_{c}}{q} \operatorname{Ln}\left(\frac{I_{p h}}{I_{0}}\right)$

$I_{s c} \approx I_{p h}$

where $A, K$, and $q$ are the diode ideality factor, Boltzmann's constant and electron charge, respectively. Moreover, $I_{0}$ relates to the current dependency on temperature and short circuit current [15].This means that by manipulating Poynting vector in the plasmonic condition, solar cell's key parameters such as optical generation rate and solar cell photon current are remarkably changed. For this, in this study, we investigated the NPs optical properties' manipulating effect on generation rate and optical power, and Poynting vector was considered as the critical factor for this study. At last, the solar cell's $I-V$ (current-voltage) relations, which depend on $I_{p h}$ and its original factors such as NPs plasmonic field intensity, are introduced by:

$V=\frac{A K T_{c}}{q} \operatorname{Ln}\left(\frac{I_{p h}-I_{0}-I}{I_{0}}\right)-I R_{s}$
$I=I_{p h}-I_{0} \exp \left[\frac{q\left(V+I R_{s}\right)}{A K T_{c}}-1\right]-\frac{\left(V+I R_{s}\right)}{R_{s h}}$

In these equations, $R_{s}$ and $R_{s h}$ are the solar cell series and shunt resistors, respectively. Therefore, with the knowledge about this point, which the NPs plasmonic field intensity has effectively influenced the photon current and solar cell output voltage and current; we can design a solar cell based on NPs or NRs for engineering the solar cell photon current. 


\section{III.SIMULATIONS RESULTS AND DISCUSSION}

In this section, the simulation results in the case of 2-D simple structure solar cell are illustrated. Based on equations 8-13, which emphasize the plasmonic effect of optical power and optical generation rate on solar cell photon current and its influence on output current and voltage; we compared three different structures, which are schematically shown in Fig. 1.
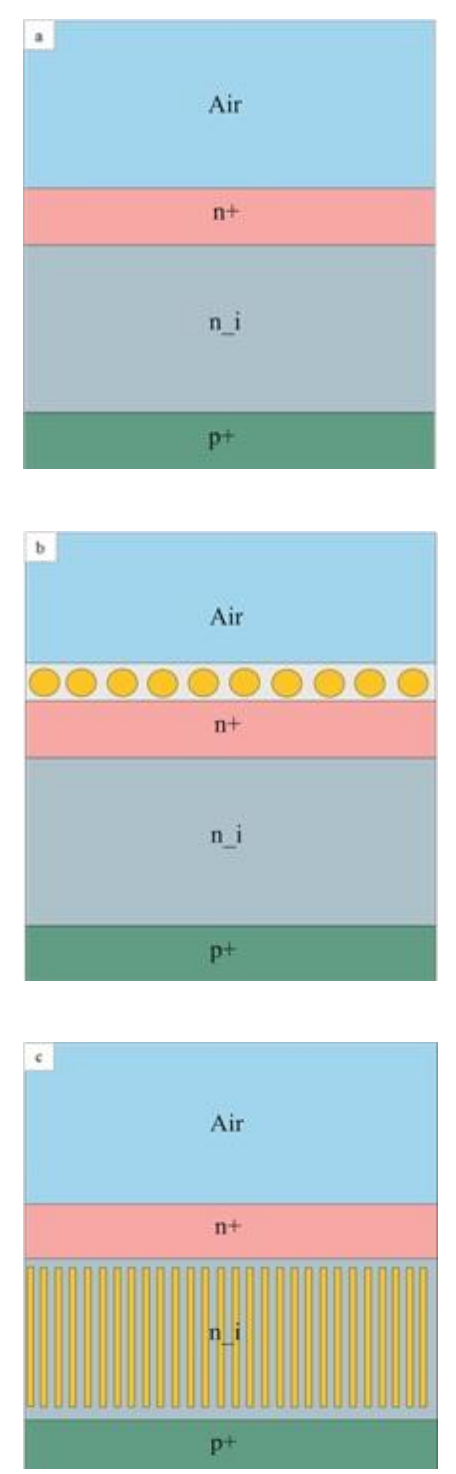

Fig. 1. Solar cell structures (a) without any NPs (traditional), (b) with NPs in the addition region, and (c) NRs in the intrinsic area"

Actually, the original aim is to manipulate the photon current based on optical power and optical generation rate engineering. In fact, by manipulating the NPs' or NRs' optical properties, we will try to alter optical power or generation rate in solar cell. Fig 1a shows the traditional simple p-n structure without any NPs or NRs. But in Figs. $1 \mathrm{~b}$ and 1c, for improvement, we use arrays of nanostructures (NPs or NRs), which severely manipulates the electromagnetic field and generation rate in different regions. In the following, by manipulating NPs' or NRs' geometry such as size, shape, material and inter-distance, the electromagnetic field and generation rate will be changed. We tried to select the better and optimum structure to have a maximum generation rate in solar cell structure. Initially, light propagation in different regions of traditional structure is illustrated in Figs. 2a and $b$. In all simulations, the incidence light is propagated in the $y$-direction with a $x$-direction polarization. In traditional structure without any NPs and NRs, initially, light propagates in the air and then enters a dense area such as silicon. So, because of the different optical properties of air and $\mathrm{Si}$, the interaction between light and materials will be changed. Based on materials' optical properties, the light propagation changes. These figures show that the light amplitude dramatically decreases when light entered the solar cell's area. With this, optical generation rate will severely decrease. It can be considered as an important factor for the low efficiency of traditional solar cell. Hence, for solving the problem, the plasmonic-based solar cells are proposed. In this one, the plasmonic NPs are used to manipulate the light interaction with matter in the solar cells regions. In other words, the near-field plasmonic resonance of NPs influences light propagation in solar cell. So, the optical generation rate is engineered. In the following, based on Fig. 2c, arrays of Au NPs are used beyond $\mathrm{Si}+$ regions and then by irradiation of light on structure in the $y$ direction and with a $\mathrm{x}$-direction polarization, the key parameters such as electric and magnetic field distribution and their gradients are altered, which are shown in Figs. 2c and $2 d$. Figure $2 c$ shows that the NPs plasmonic affects light propagation in solar cell's different regions. For better illustration you 
can see the alteration of electric field profile as 1-D in Fig. 2c .
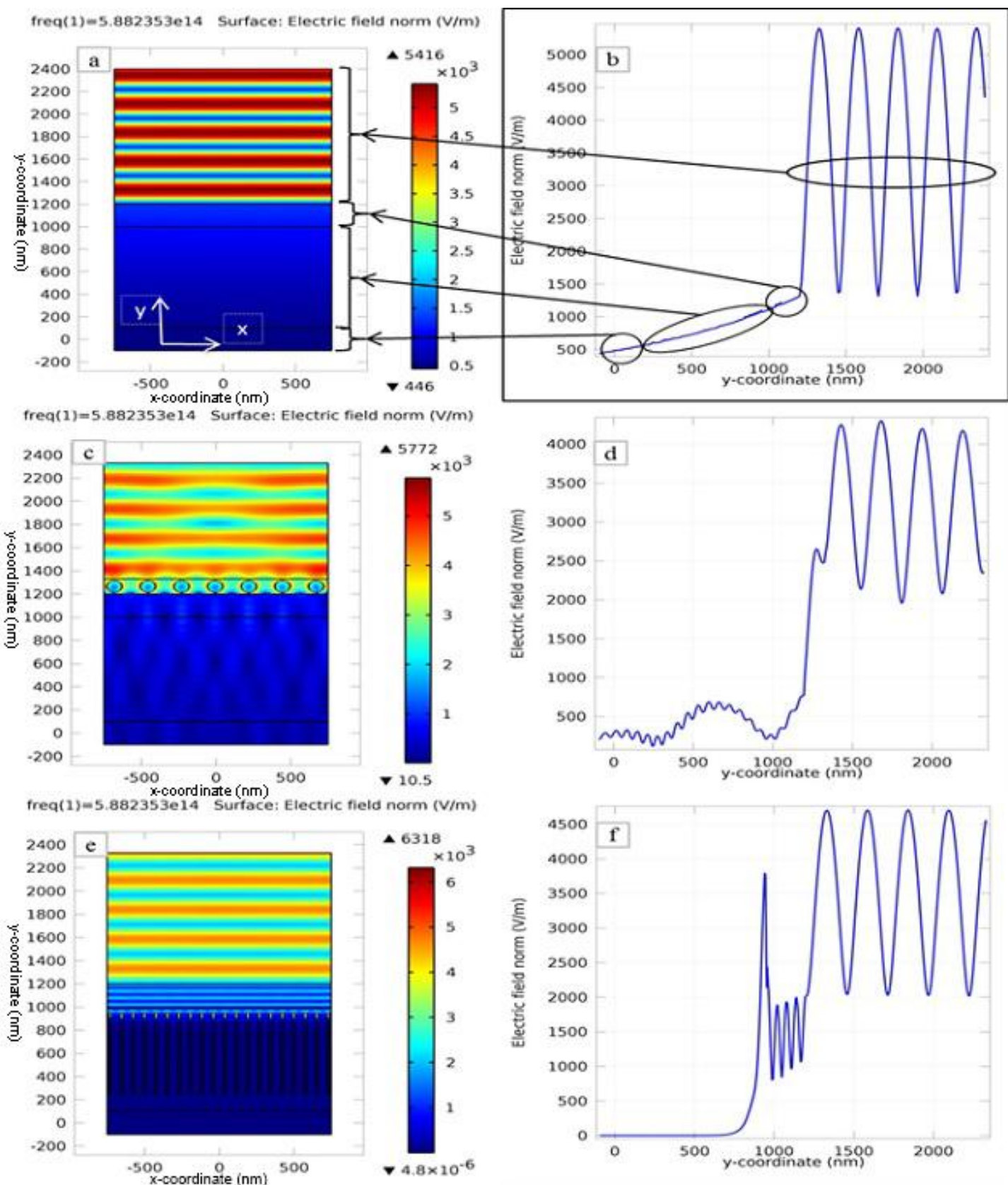

Fig. 2. Propagation of light in the (a) and (b) traditional structure, (c) and (d) improved structure with NPs in the addition region, (e) and (f) improved structure with NRs in the intrinsic area".

It means that with plasmonic state and nonuniform electromagnetic field production, generation rate dramatically changes. It is because of the influence of Au NPs near-field effect on external applied field. In another simulation, arrays of NRs were used in the intrinsic region. It is shown that using NRs in the intrinsic area dramatically affects electromagnetic distribution profile and so optical power and generation rates are changed. This is contributed to the NRs plasmonic effect in the intrinsic area; because NRs create near-field influence on original field and disturbs it. It is better to compare the NPs and NRs effect on electrical field profile in the different area of solar cells, which are shown in Figs. $2 d$ and $2 f$. It is because in the following, we can easily manipulate the key parameters and select the optimum structures. For this, NPs and NRs parameters such as their size, inter-distance and materials effects are perused in Figs. 3 and 4, respectively. 


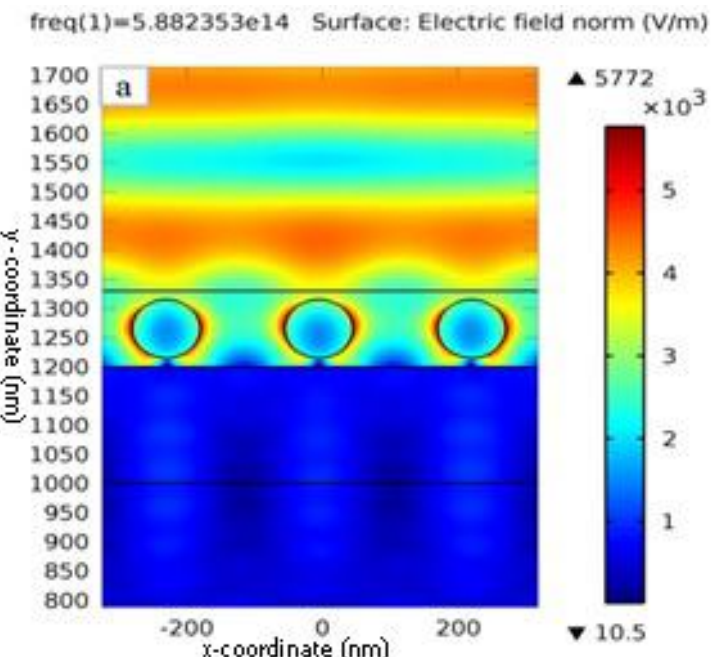

freq(1) $=5.882353 \mathrm{e} 14$ Surface: Electric field norm $(\mathrm{V} / \mathrm{m})$

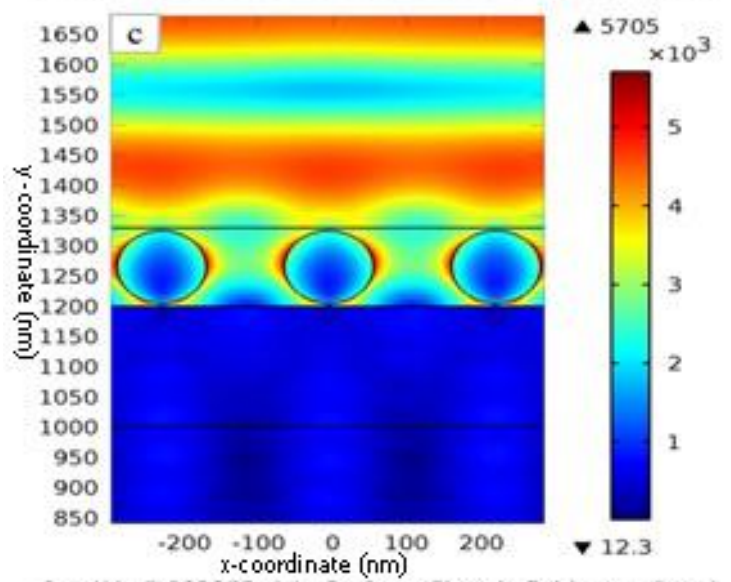

freq $(1)=5.882353 \mathrm{e} 14$ Surface: Electric field norm $(\mathrm{V} / \mathrm{m})$

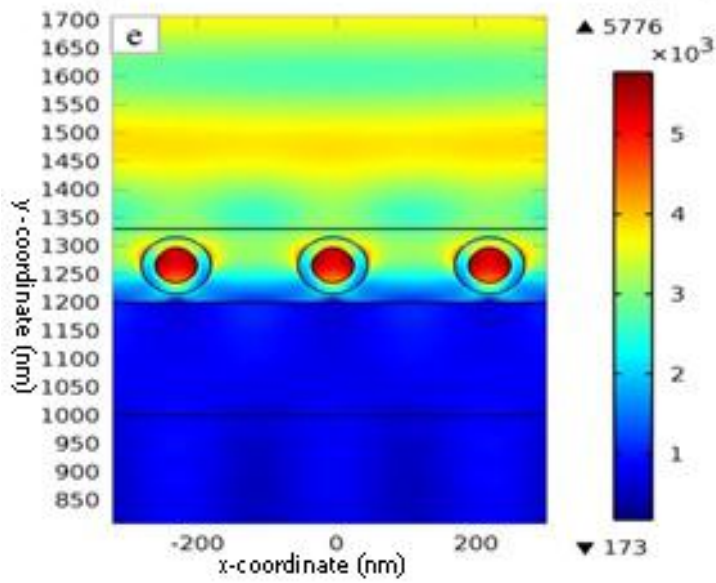

freq(1) $=5.882353 \mathrm{e} 14$ Surface: Electric field norm $(\mathrm{V} / \mathrm{m})$

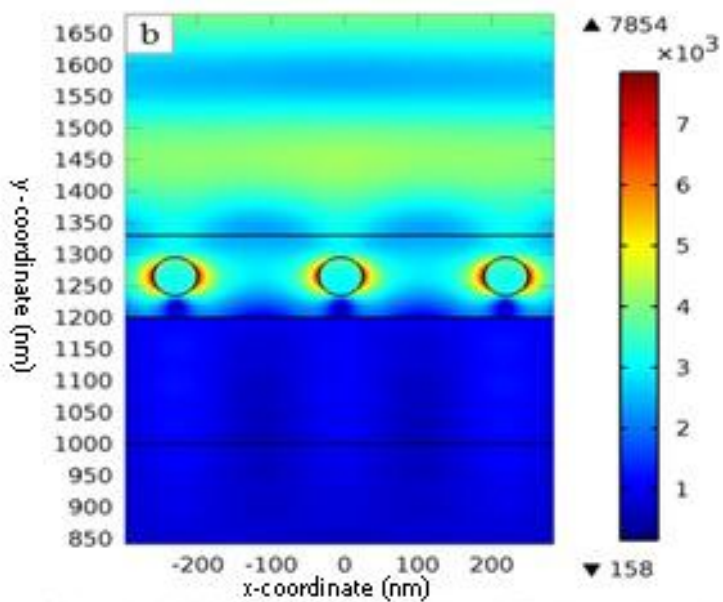

freq $(1)=5.882353 \mathrm{e} 14$ Surface: Electric field norm (V/m)

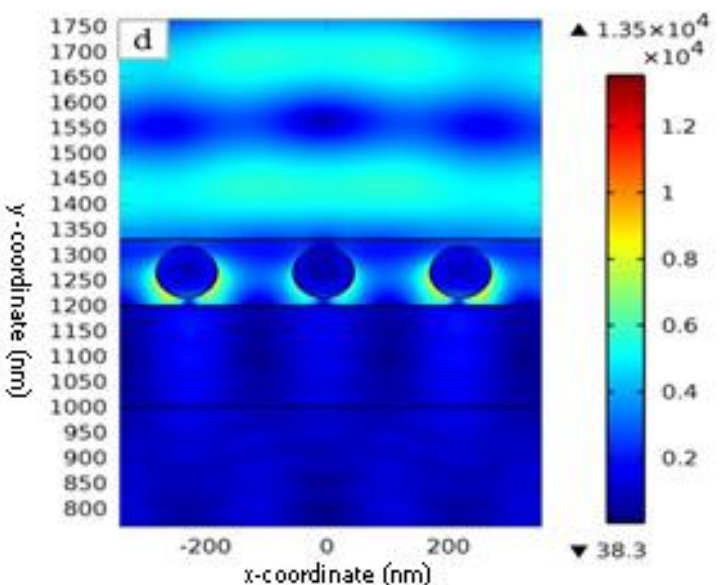

freq $(1)=3.649635$ e14 Surface: Electric field norm $(\mathrm{V} / \mathrm{m})$

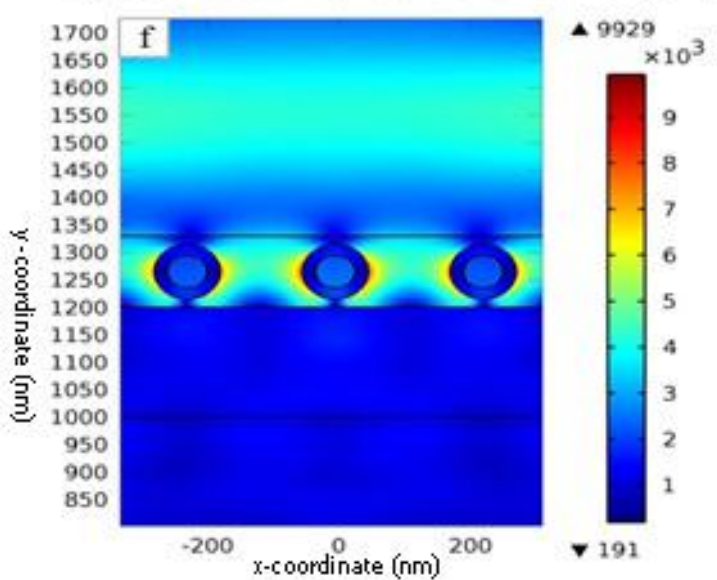

Fig. 3. Influence of NPs plasmonic resonances on light propagation in the structure, (a) NPs radius $50 \mathrm{~nm}$, (b) NPs radius $30 \mathrm{~nm}$ and increasing of NPs inter-distance, (c) NPs radius $60 \mathrm{~nm}$ and decreasing of NPs inter-distance, (d) NPs material Ag, (e) core/shell NPs at $517 \mathrm{~nm}$, and (f) core/shell NPs at $833 \mathrm{~nm}$.

Figure 3 shows NPs parameters' effect on electrical profile distribution in the solar cell's different areas. In this figure, the influence of NPs plasmonic resonances on light propagation is shown: [(a) NPs radius $50 \mathrm{~nm}$, (b) NPs radius $30 \mathrm{~nm}$ and increasing NPs' inter-distance, (c) NPs radius $60 \mathrm{~nm}$ and decreasing NPs inter-distance, (d) NPs material Ag, (e) core/shell NPs at incidence wavelength $517 \mathrm{~nm}$; f) core/shell NPs at incidence wavelength $833 \mathrm{~nm}$.] 

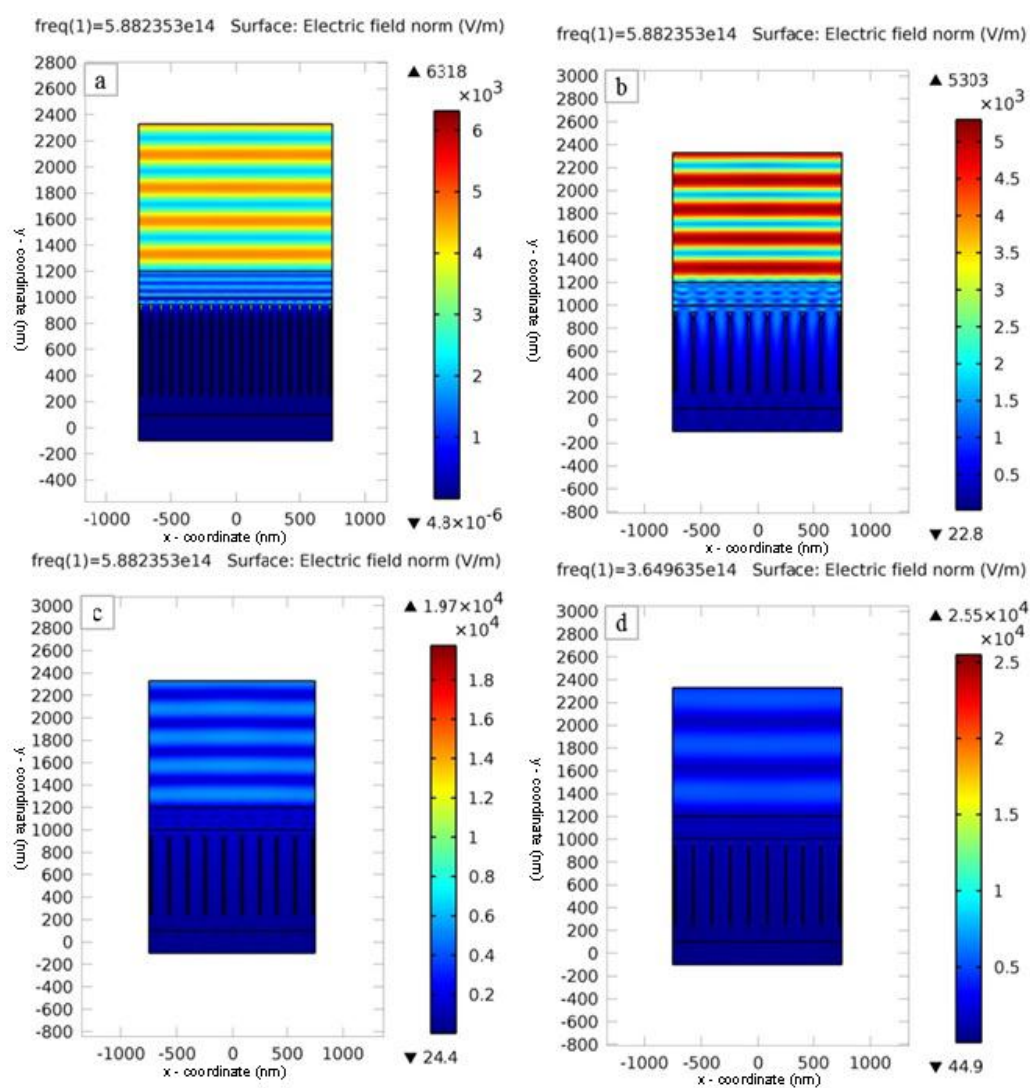

Fig. 4. Influence of NRs plasmonic resonances on light propagation in the structure, (a) NRs inter-distance $40 \mathrm{~nm}$, (b) NRs inter-distance $80 \mathrm{~nm}$, (c) NRs material Ag, (d) wavelength effect $833 \mathrm{~nm}$.
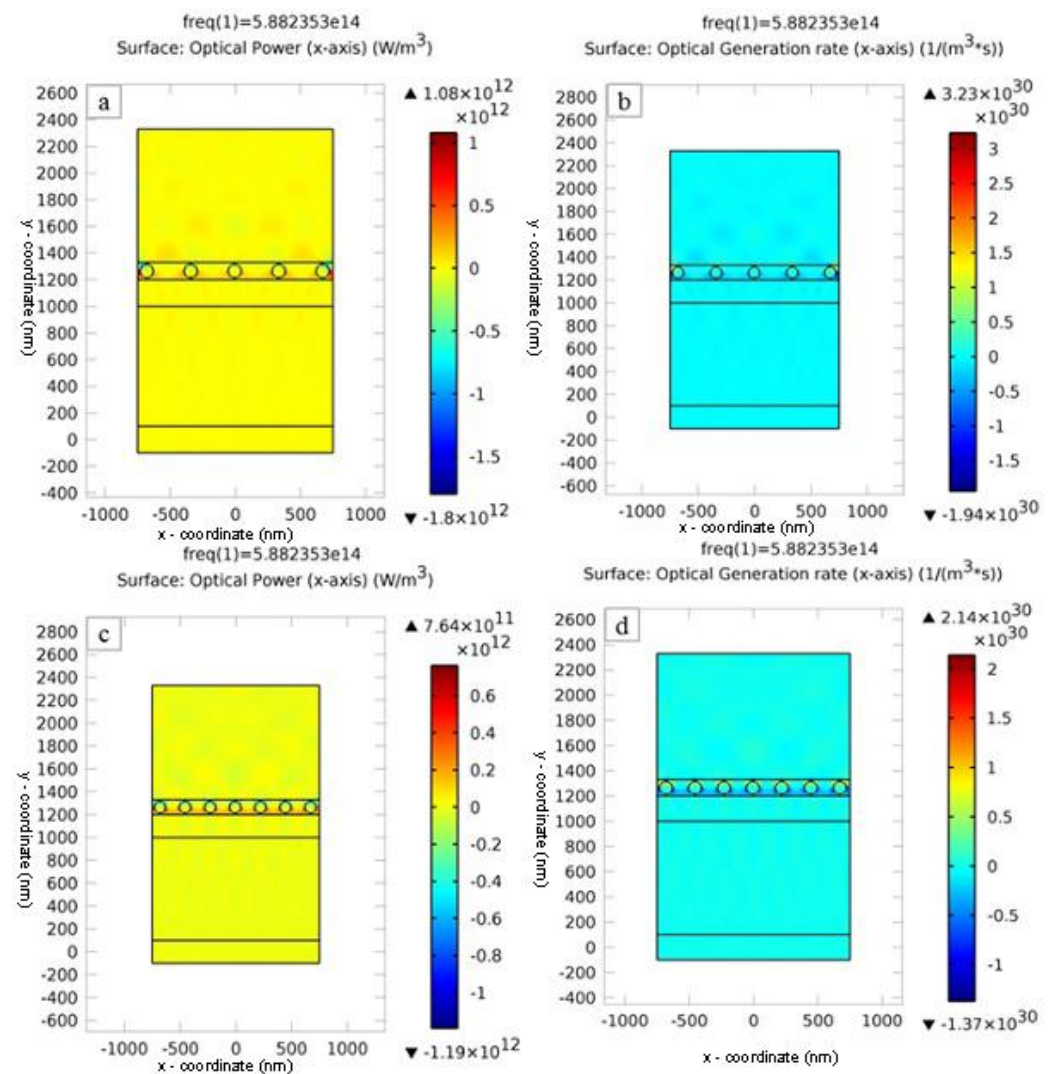

Fig. 5 NPs influence on optical power and generation rate, (a) NPs size alteration effect, (b) NPs materials effect, (c) NRs inter-distance changing effect, and (d) NRs materials effect". 

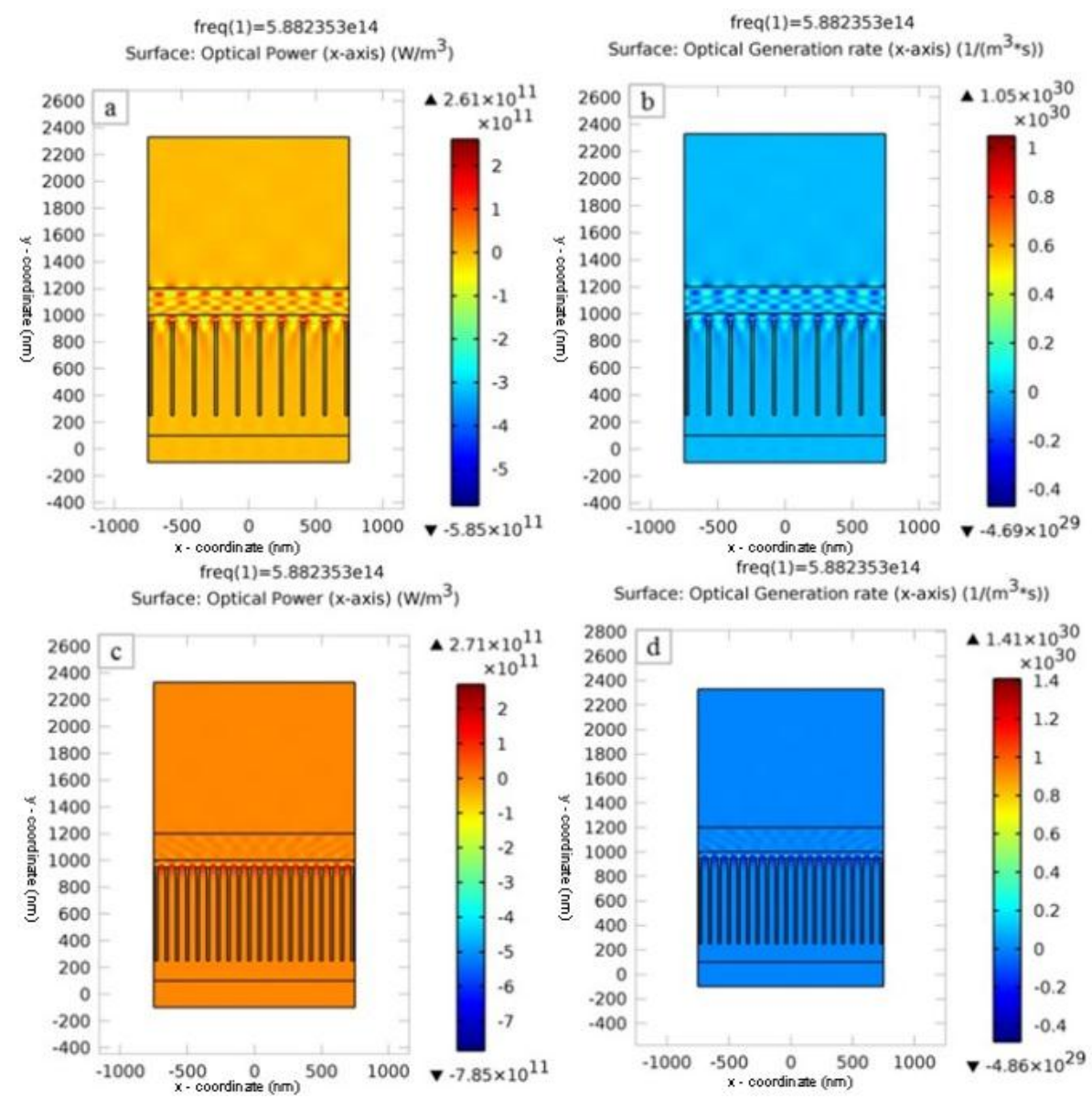

Fig. 6. NRs influence on optical power and generation rate, (a) NPs size alteration effect, (b) NPs materials effect, (c) NRs inter-distance changing effect, and (d) NRs materials effect.

It should be noted that with increasing the NPs' radius, the inter-distance between NPs is decreased, so we can easily see the plasmonicplasmonic interaction between the two adjacent NPs. It is a good case for manipulating near-filed effect and influence the external field. Moreover, the effect of NPs material on their plasmonic resonance was discussed. In this case, we used Ag rather than $\mathrm{Au}$. It is shown that because of distance of $\mathrm{Ag}$ plasmonic resonance from incidence wavelength $510 \mathrm{~nm}$, in this work, the present format of NPs doesn't show any strong plasmonic resonance in this wavelength, which is shown in Fig. 3d.

Moreover, we examined the core/shell NPs such as $\mathrm{SiO}_{2} / \mathrm{Au}$ for extra engineering of the near-field effect. It is because in this case we easily manipulated the plasmonic interaction between inner- and outer interface of Au shell.
In other word, by controlling the $\mathrm{Au}$ shell thickness, the plasmonic interaction is changed. But, for core/shell NPs only the wavelength effect was perused, which are shown in Figs. 3e and 3f. In the incidence wavelength around $517 \mathrm{~nm}$, Fig. 3e, innerinterface plasmonic effect is dominant and this case is not useful for solar cell application. It means that this incidence wavelength couldn't excite $\mathrm{Au}$ shell properly. It is because introducing resonance in the core region causes heat generation in NPs and leads to their deformation. But, in the case of $833 \mathrm{~nm}$, the core/shell NPs reveal a good plasmonic effect and severely influence external field. It is due to this fact that $\mathrm{Au}$ shell shows plasmonic resonance around 750-850 $\mathrm{nm}$. In another try, we perused the NRs parameters' effect on electrical profile variation. 
The influence of NRs plasmonic resonances on light propagation is shown in Fig. 4: [(a) NRs inter-distance $40 \mathrm{~nm}$; (b) NRs inter-distance 80 nm, (c) NRs material Ag, (d) wavelength effect $833 \mathrm{~nm}$.] It is easily seen that with altering NRs inter-distance, which are shown in Figs. 4a and b, the interaction between NRs adjacent are severely altered and affect field profile in solar cell region. Moreover, the effect of NRs materials and incidence wavelength are investigated and their simulation results are illustrated in Figs. 4c and d, respectively. Finally, our investigations are associated to the optical power and its related optical generation rate. For this, the effect of NPs and NRs' size, materials and inter-distance are studied. Their related simulation results are shown in Figs. 5 and 6.

It is shown that the NRs effect on optical power and optical generation rate is higher than NPs. These figures show that the carrier generation in the solar cell equations (7 and 8) is handled by NRs and NPs plasmonic resonance. It is our article's original aim and the improved structure for better manipulation can be considered in Fig. $6 b[1,13]$. It is better to mention that we can use the NPs in the intrinsic area and maybe its effect is better than NRs in this region.

\section{IV.CONCLUSIONS}

In this study, it was shown that the NPs generating near-field electromagnetic wave severely affects optical power and generation rate, which handles the carrier's generation in the solar cell equations. In other words, by manipulating plasmonic properties of NPs or NRs in solar cells structure, distribution of electromagnetic fields is altered. The simulation results showed that for improving the generation rate, as an important parameter in solar cells, the alteration of NPs' or NRs' materials, shapes, inter-distance and medium material, are necessary.

\section{REFERENCES}

[1] S. Pillai, K.R. Catchpole, T. Trupke, and M.A. Green, "Surface Plasmon enhanced silicon solar cells," J. Appl. Phys. Vol. 101, pp. 093105 (1-8), 2007.

[2] J.O. Schumacher, Numerical simulation of silicon solar cells with novel cell structure, $\mathrm{PhD}$ thesis, University of Konstanz, Germany, 2000.

[3] P. Spinelli, V.E. Ferry, J. Groep, M. Lare, M.A. Verschuuren, R.E.I. Schropp, H.A. Atwater, and A. Polman, "Plasmonic light trapping in thin-film $\mathrm{Si}$ solar cells," IOP Science, Vol. 14, pp. 024002 (1-11), 2012.

[4] J. Jung, T. Søndergaard, T.G. Pedersen, K. Pedersen, A.N. Larsen, and B.B. Nielsen, "Dyadic Green's functions of thin films: Applications within plasmonic solar cells," Phys. Rev. Vol. 83, pp. 085419 (1-14), 2011.

[5] S. Tembhurne, M. Dumortier, and S. Haussener, "Heat transfer modeling in integrated photo electrochemical hydrogen generators using concentrated irradiation," IEEE Proc. of the 15th International Heat Transfer Conference, Aug. 10-15, 2014, Kyoto, Japan.

[6] J. Yang, J. You, C. Chen, W. Hsu, H. Tan, X.W. Zhang, Z. Hong, and Y. Yang, "Plasmonic Polymer Tandem Solar Cell," ACSNANO Amer. Chem. Soc. Vol. 5, pp. 6210-6217, 2011.

[7] M.A. Green and S. Pillai, "Harnessing plasmonic for solar cells," Nature photonics, Vol. 6, pp. 130 132, 2012.

[8] V.E. Ferry, L.A. Sweatlock, D. Pacifici, and H.A. Atwater, "Plasmonic nanostructure design for efficient Light coupling into solar cells," Nanolett. Vol. 8, pp. 4391-4397, 2008.

[9] R. Guo, H. Huang, P. Chang, L. Lu, X. Chen, X. Yang, Z. Fand, B. Zhu, and D. Li, "Coupled optical and electrical modeling of thin-film amorphous silicon solar cells based on nanodent plasmonic substrates," Nano Energy, Vol. 8, pp. 141-149, 2014.

[10] Y.A. Akimov, W.S. Koh, and K. Ostrikov, "Enhancement of optical absorption in thinfilm solar cells through the excitation of higher-order nanoparticle Plasmon modes," Opt. Express, Vol. 17, pp. 10195-10205, 2009.

[11] S.D. Standridge, G.C. Schatz, and J.T. Hupp, "Toward plasmonic Solar Cells: Protection of Silver Nanoparticles via Atomic Layer Deposition of $\mathrm{TiO}_{2}$," Langmuir, Vol. 25, pp. 2596-2600, 2009. 
[12] W. Jiang, D.S. Ginger, M. Salvador, and S.T. Dunham, "Optics and Device Simulation of Surface plasmonic Enhancement of Organic Solar Cell Performance using Silver NanoPrisms," SISPAD, September 5-7, Denver, CO, USA, pp. 245-248, 2012.

[13]T.F. Villesen, On the Enhancement of Light Absorption in Si Solar Cells by Front-Side plasmonic Scattering, $\mathrm{PhD}$ Thesis science and technology Aarhus University, 2014.

[14] Y.P. Singh, A. Kumar, A. Jain, and A. Kapoor, "Enhancement in Optical Absorption of plasmonic Solar Cells," Open Renew. Energy J. Vol. 6, pp. 1-6, 2013.

[15]S.S. Verma, "Plasmonic solar cells," Government of India Ministry of New and Renewable Energy AKSHAY URJA, Vol. 7, pp. 22-26, 2013.

[16] K.R. Catchpole and A. Polman, "Plasmonic solar cells," Opt. Express, Vol. 16, pp. 2179321800, 2008.

[17]U.W. Paetzold, K. Bittkau, M. Meier, R. Carius, and U. Rau, "Simulation-Based Analysis of plasmonic Light Trapping in ThinFilm Silicon Solar Cells," 13th International Conference on Numerical Simulation of Optoelectronic Devices (NUSOD), Vol. 19, pp. 99-100, 2013.

[18]M.B. Eteiba, E.T. El Shenawy, J.H. Shazly, A.Z. Hafez, "A Photovoltaic (Cell, Module, Array) Simulation and Monitoring Model using MATLAB ${ }^{\circ / G U I}$ Interface," Int. J. Computer Appl. Vol. 69, pp. 14-28, 2013.

[19] T. Salmi, M. Bouzguenda, A. Gastli, and A. Masmoudi, "MATLAB/Simulink Based Modeling of Solar Photovoltaic Cell," Int. J. Renew. Energy Research-IJRER, Vol. 2, pp. 213-218, 2012.

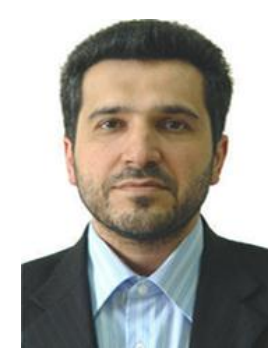

Farshad Farhadnia, He received his MSc. degree from Electrical and Computer
Engineering from University of Tabriz, Iran in 2006. He worked for his M.Sc. thesis on the Multi agent systems. He is now PHD student and works on Solar cell based on Nano particle and plasmonics properties.

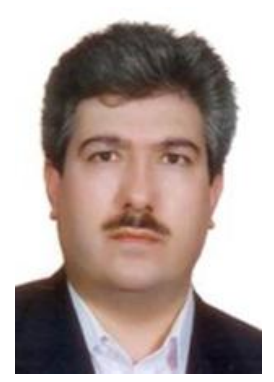

Ali Rostami, received the Ph.D. degree in Electrical and Computer Engineering from Amirkabir University of Technology, Tehran, Iran in 1998. He is currently a professor of Faculty of Electrical and Computer Engineering, Photonics and Nano crystal Research Lab, University of Tabriz, Iran. He has worked on the research fields of Nano photonics, Opt mechatronics, plasmonics, Photonic Crystals (Modeling and Optical VLSI), Optical Processing and Computing and etc.

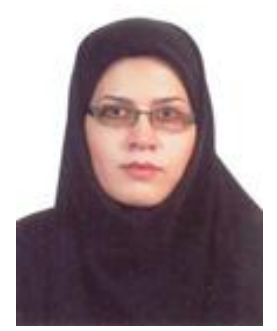

Samieh Matloub, received her Ph.D. in photonic/electronic engineering from University of Tabriz, Tabriz, Iran, in 2010. She is currently Assistant professor of electronic engineering and photonics science at the University of Tabriz. Her teaching and research interests include optoelectronic devices and optical integrated circuits. 